\title{
Impact of Spectral Filtering on Multi-pulsing Instability in Mode-locked Fiber Lasers
}

\author{
Xianting Zhang, Feng Li, K. Nakkeeran, Jinhui Yuan, Member, IEEE, Zhe Kang, J. Nathan Kutz, and \\ P. K. A. Wai, Fellow, IEEE
}

\begin{abstract}
We investigate the impact of spectral filtering in mode-locked fiber lasers with an extended geometrical model. Our iterative model, which includes gain, loss and the pulse shaping effects of chromatic dispersion and selfphase modulation, is used to model the laser cavity dynamics. Simulations show that broadband pulses experience large losses from spectral filtering in the cavity, leading to a number of potential laser instabilities and outcomes such as multi-pulsing, periodic and chaotic states, or a single pulse which transitions to a higher energy state. For narrow band spectral filtering, the laser dynamics is dominated by the gain-loss dynamics in the cavity which causes multi-pulsing. For broadband spectral filtering, the nonlinearity induced spectral reshaping of the single pulse can lead to a discontinuous pulse energy transition that circumvents multi-pulsing. The inclusion of third order dispersion shows that the multi-pulsing instability is induced even in the case of broadband spectral filtering.
\end{abstract}

Index Terms-Mode-locked fiber lasers, spectral filtering, multi-pulsing, nonlinear dynamics.

\section{INTRODUCTION}

$\mathrm{P}$ assively mode-locked fiber lasers have been extensively investigated in the past decades because of their broad range of applications in spectroscopy, optical sensing, supercontinuum generation, frequency comb generation, etc. The robust generation of high-energy, ultrashort pulses is of critical importance for most of these applications. Based on various mode-locking techniques, e.g. nonlinear polarization rotation (NPR) [1], semiconductor saturable absorber mirrors (SESAMs) [2] and nonlinear optical loop mirrors (NOLMs) [3, 4], ultrashort pulses with a duration of $\sim 20$ fs and single pulse energies of more than $10 \mathrm{~nJ}$ can be generated from single mode fiber laser cavities. Performance gains for such lasers is

This work was partially supported by the National Natural Science Foundation of China (61475131), Research Grant Council of the Hong Kong SAR (PolyU152144/15E), Shenzhen Science and Technology Innovation Commission (JCYJ20160331141313917) and the Hong Kong Polytechnic University (1-ZVGB). X. Zhang and F. Li contributed equally to this paper. (Corresponding authors: Feng Li and Jinhui Yuan).

X. Zhang, F. Li, Z. Kang, and P. K. A. Wai are with the Photonics Research Centre, Department of Electronic and Information Engineering, The Hong Kong Polytechnic University, Hung Hom, Hong Kong SAR, and also with The Hong Kong Polytechnic University Shenzhen Research Institute, Shenzhen 518057, China. (e-mail: enlf@polyu.edu.hk). impeded by the intrinsic multi-pulsing instability [5-8]. This ubiquitous phenomenon sets a critical limitation on achievable pulse energies in a mode-locked fiber laser cavity. In order to overcome the performance limitations, the mechanisms leading to the multi-pulsing instability need to be circumvented.

The multi-pulsing instability in laser cavities has been investigated both theoretically and experimentally [9-13]. A simple geometrical model of the laser cavity that includes only the gain and nonlinear loss shows multi-pulsing is induced if the response function of the nonlinear loss, or saturable absorber (SA), is non-monotonic $[9,10]$. Such insights allow one to engineer NPR and NOLM based mode-locked lasers, both of which have non-monotonic SA, with an increased threshold for the multi-pulsing instability [11, 12]. The geometrical model [9] also suggests that in the absence of pulse shaping, multi-pulsing can be avoided altogether if the response function of the SA is monotonic. However, multi-pulsing is still observed in mode-locked Ti:sapphire lasers with semiconductor SA, which has a monotonic response function [13]. Thus, the pulse shaping and/or the pulse evolution in the cavity, which are not included in the geometrical model, need to be accounted for in order to characterize the multi-pulsing mechanism. A typical mode-locked fiber laser cavity consists of a gain element, a section of nonlinear and dispersive fiber, an output coupler, saturable absorption, and spectral filtering. The bandwidth and shape of the spectral filter play a critical role in modifying the pulse in the spectral domain. Broadband pulses experience larger losses due to the spectral filter. Thus pulses with different energies (and associated bandwidths) experience an energy dependent loss even though the filtering action is only a frequency dependent $[13,14]$.

The impact of spectral filtering on mode-locked fiber lasers is well known [13-16]. When a narrow-band spectral filter is used in a normally dispersive fiber cavity, self-similar pulse propagation in the gain fiber greatly enhances the pulse quality [17], but with spectral filtering remaining a trigger for the multi-

K. Nakkeeran is with the School of Engineering, Fraser Noble Building, University of Aberdeen, Aberdeen AB24 3UE, United Kingdom.

J. Yuan is with The Hong Kong Polytechnic University Shenzhen Research Institute, Shenzhen 518057, China, and also with the State Key Laboratory of Information Photonics and Optical Communications, Beijing University of Posts and Telecommunications, Beijing, 100876, China. (e-mail: yuanjinhui81@163.com).

J. N. Kutz is with the Department of Applied Mathematics, University of Washington, Seattle, WA 98195-2420, USA. 
pulsing instability [14]. The pulse evolution in an anomalously dispersive fiber laser cavity is more complex, and hence periodic and chaotic dynamics are observed in addition to the multi-pulsing transition [18-20]. In such cavities, SA narrows the pulse and hence broadens its spectrum. Thus the spectrally broadened pulse is narrowed by the action of the spectral filter. So in addition to balancing the effects of anomalous dispersion and self-phase modulation, the mode-locked pulse must also balance the physics of SA and spectral filter [21]. Thus a theoretical model must account for both the balances of the soliton evolution and the dissipative spectral filtering action.

In this paper, we investigate the effects of the spectral filter on the laser dynamics by extending the geometrical model [9, 10] to include the pulse shaping effects of chromatic dispersion and self-phase modulation. In particular, we explicitly characterize the filtering action imposed by the bandpass filter in the cavity. We show that our extended geometrical model is well suited for systematically investigating the nonlinear dynamics of the pulse evolution which includes multi-pulsing, periodic and chaotic states and single pulse high-energy transition in mode-locked fiber laser cavities.

\section{TheoreticAl Model AND EXTENDED GeOMETRICAL DESCRIPTION}

\section{A. Combined action of SA and spectral filter}

In the current geometrical model, the multi-pulsing dynamics, which includes bifurcations and chaos, is only observed in a cavity with a non-monotonic response of SA [9, 10]. But in most laser cavities, the pulse temporal and spectral profiles undergo significant changes when the pump power is varied. Specifically, pulses with different spectral profiles undergo different filtering losses. The action of the spectral filter must then be included in the existing geometrical model for a better description of the mode-locking process.

Although the mode-locked pulses in an anomalously dispersive fiber laser cavity are not solitons, they retain some properties of solitons. Thus the area theorem developed for solitons in passive fibers can be used to qualitatively describe the connection between the pulse duration, bandwidth and energy. As per the area theorem, the product of the soliton duration $\tau$ and peak amplitude $A_{0}$ is a constant. Hence the pulse duration $\tau$ is related to the pulse energy as $E \propto \tau A_{0}^{2}$. For a chirp free soliton, the bandwidth is inversely proportional to the pulse duration $\tau$. So the bandwidth is proportional to the pulse energy $E$. The orange dash-dotted curve in Fig. 1 shows the energy transmittance of a Gaussian spectral filter for solitons with different energies. The transmittance decreases monotonically as the pulse energy increases and hence increasing the bandwidth of the pulse. The blue dashed curve in Fig. 1 shows the monotonically increasing response of a SA given by $T=1-0.85 /\left(1+P(t) / P_{\text {sat }}\right)$, where $P(t)$ is the temporal power profile of the pulse and $P_{\text {sat }}$ is the saturation power of the SA. The red solid curve in Fig. 1 shows the combined transmission response of the SA, and spectral filter is no longer monotonic, but rises to a maximum and then decreases monotonically.
According to the geometrical model $[9,10]$, when the combined transmission response of the SA and spectral filter is dropped to the level of small signal, further increase in the cavity energy will trigger the multi-pulsing instability.

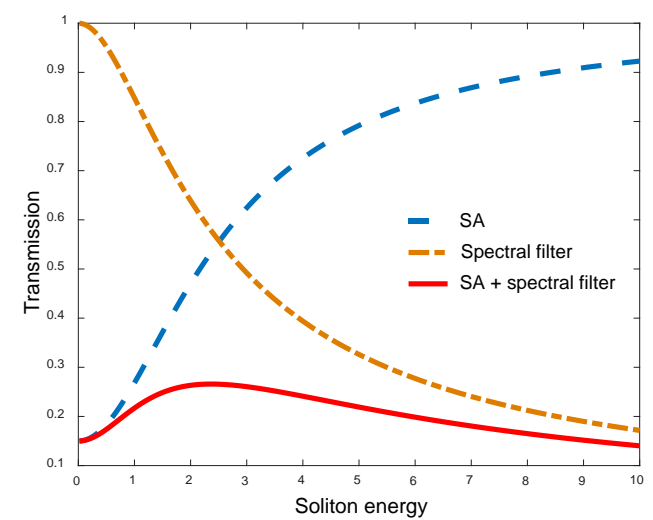

Fig. 1. Transmission response of the SA, filter and their combination versus soliton energy.

However, the dynamics of the soliton in mode-locked fiber lasers is more complicated than described by Fig. 1. Because of the gain in the laser cavity, the pulses cannot be maintained as a fundamental soliton and can evolve to higher order solitons during the propagation. Such dynamics must also be modeled by a governing equation for the cavity that includes the pulse shaping due to the nonlinear gain fiber, SA and the spectral filter.

\section{B. Governing equation of the cavity}

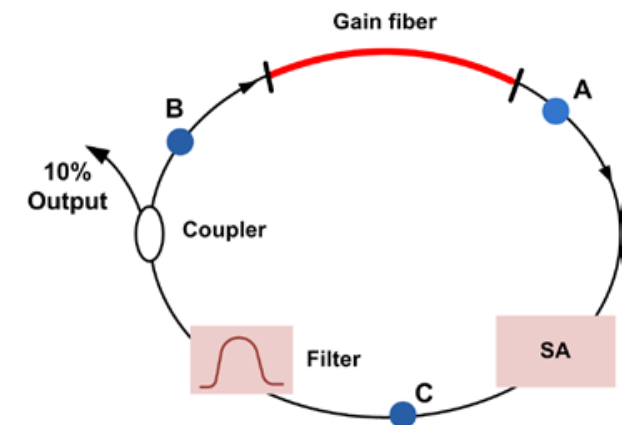

Fig. 2. Schematic of a typical mode-locked fiber laser cavity that consists of a section of gain fiber, an SA, a spectral filter and an output coupler.

Figure 2 shows the schematic of a typical mode-locked fiber laser cavity. In the cavity, the gain fiber provides pulse amplification. The SA provides the nonlinear loss that triggers the mode-locking mechanism. The spectral filter provides the frequency dependent loss that controls the spectral bandwidth of the pulse in the cavity. The input points of the gain fiber, SA and spectral filter are marked as A, B, and C respectively as references for the following discussion.

As this study is primarily focused on the lumped point action of the spectral filter in the cavity, distributed models such as Haus' master equation and complex Ginzburg Landau equation [22-26], cannot be used for such investigations. In a modelocked fiber laser cavity with a strong spectral filtering, the variations in pulse evolution can be significant for high-energy pulses [17, 21]. To capture the effects of the spectral filter and 
SA, we use a discrete model in which the optical components in the cavity are modeled separately. An averaged gain fiber model is adopted to capture the pulse dynamics in the fiber, where the effect of the gain, dispersion and nonlinearity are all appropriatively included with their respective parametric values. Although such fiber model cannot accurately simulate the pulse evolution in different fibers, it has captured the most dominant effects. The averaged model for the fiber will greatly simplify the whole model for the laser cavity that in turn will help to investigate the spectral filtering action on the pulse dynamics. In the discrete model, the pulse is modified by every optical component successively in each round trip of the laser cavity.

The pulse evolution in the gain fiber of the laser cavity is modeled by the nonlinear Schrödinger equation (NLS) with gain as [14],

$$
\frac{\partial A}{\partial z}=\frac{1}{2} g(z) A-\frac{i}{2} \beta_{2} \frac{\partial^{2} A}{\partial t^{2}}+\frac{\beta_{3}}{6} \frac{\partial^{3} A}{\partial t^{3}}+i \gamma|A|^{2} A,
$$

where $\beta_{2}, \beta_{3}$ and $\gamma$ are the second order dispersion, third order dispersion, and nonlinear coefficients, respectively. We focus on fiber lasers in the anomalous dispersion regime since these are more common. The gain coefficient $g(z)$ is updated dynamically in the propagation by $[9,10,14]$

$$
g(z)=\frac{g_{0}}{1+\int|A|^{2} d t / E_{\mathrm{s}}},
$$

where $g_{0}$ is the small signal gain coefficient and $E_{\mathrm{s}}$ is the saturation energy. In experiments, the increase of pump power increases $g_{0}$ and $E_{\mathrm{s}}$ simultaneously [9, 10,14], but the variation of $E_{\mathrm{s}}$ is enough to capture most of the dynamics. . In this study we purposely used a flat gain profile to avoid the conflict between the gain profile and filter profile. Considering flat gain profile will help to correctly investigate the action of filter in the laser dynamics with respect to the profile and bandwidth of the filter.

The SA is modeled by a power dependent transmittance function $[14,16]$

$$
T_{S A}(t)=1-\frac{l_{0}}{1+|A(t)|^{2} / P_{\text {sat }}},
$$

where $P_{\text {sat }}$ is the saturation power and $l_{0}$ is the unsaturated loss of the SA.

A Gaussian bandpass filter is modeled in the frequency domain as

$$
H_{f}(\omega)=H_{0} \exp \left(-\omega^{2} / \omega_{b}^{2}\right),
$$

where $\omega_{b}$ is the filter bandwidth and $H_{0}$ is the peak transmittance of the filter. With this Gaussian profile for the bandpass filter, it can be considered that the combined action of both gain and filter is having a Gaussian profile for a realistic situation for the fiber gain. In other words we can say that both the fiber gain and the spectral filter has the same Gaussian profile. In simulations, the coupling loss of the output coupler is included in the filter model by multiplying an appropriate linear scaling factor to the peak transmittance $H_{0}$.

To model the dynamics of mode-locked fiber lasers, generally we normalize the governing equation of the propagation to

$$
\frac{\partial U}{\partial \xi}=\frac{i}{2} \frac{\partial^{2} U}{\partial \tau^{2}}+D_{3} \frac{\partial^{3} U}{\partial \tau^{3}}+i|U|^{2} U+\frac{g_{n}}{2} U,
$$

where the normalized variables are

$$
\begin{aligned}
& U=A / \sqrt{P_{\text {sat }}}, \\
& \xi=z / L_{N}, L_{N}=1 /\left(\gamma P_{\text {sat }}\right), \\
& \tau=t \times\left|\beta_{2} L_{N}\right|^{-1 / 2}, \\
& D_{3}=\frac{1}{6} \beta_{3}\left|\beta_{2}^{3} L_{N}\right|^{-1 / 2}, \\
& g_{n}=\frac{g_{0} L_{N}}{1+\int|U|^{2} d \tau / E_{\text {sat }}}, \\
& E_{\text {sat }}=E_{s} P_{\text {sat }}^{-1}\left|\beta_{2} L_{N}\right|^{-1 / 2} .
\end{aligned}
$$

The transfer functions of SA and filter are also normalized to

$$
T_{S A, n}=1-\frac{l_{0}}{1+P(\tau)},
$$

and

$$
H(\Omega)=H_{0} \exp \left[-\frac{1}{2}\left(\Omega / \Omega_{b}\right)^{2}\right],
$$

where $\Omega=\omega \times\left|\beta_{2} / \gamma P_{\text {sat }}\right|^{1 / 2}$ is the normalized angular frequency and $\Omega_{b}=2 \pi \delta_{f}$ is the normalized filter bandwidth.

The discrete model equations (5), (7) and (8) comprehensively describe the laser dynamics. Importantly, key additional physics contributions are posited in order to extended the geometrical model. In the following simulations, the gain fiber, SA and filter are modeled consecutively and iteratively until the signal in the cavity is stabilized (except for the chaotic states). During the pulse evolution in the cavity, it is difficlt to directly calculate the energy loss caused by the SA and spectral filter without knowledge of the pulse profile. But during the propagation, the pulse profile can be used to estimate the loss. By calculating the energy loss due to the SA and spectral filter, we can extend the geometrical model and interpret the nonlinear dynamics of the pulse in the cavity.

\section{SOliton Dynamics IN THE CAVITY}

In experiments, the nonlinear dynamics of the pulse, including multi-pulsing and other chaotic states, are usually observed with the increase of the pump power. To characterize the pulse dynamics in the cavity, we implement the increase of the pump power by varying the saturation energy $E_{\text {sat }}$ in the simulations. For a given set of parameters for the cavity, the signal starts from white noise and a stable mode-locked pulse is obtained with a relatively small $E_{\text {sat. }}$ Equation (5) is solved numerically using the split-step Fourier method. Then the transfer functions (7) and (8) are applied to the signal consecutively to simulate the point actions of the SA and filter. The simulation is terminated either when a stable solution is reached or after 20,000 round trips when the laser system does not converge to a stable solution. In every single simulation, $E_{\text {sat }}$ 
is kept constant. Once the final condition of the system is arrived, $E_{\text {sat }}$ is then varied from the previous value of the $E_{\text {sat }}$. The solution obtained with the former $E_{\text {sat }}$ is used as the seed for the next simulation with the new $E_{\text {sat }}$ to implement the realistic tuning process of the laser system. In simulations, the parametric values used are $g_{0}=2.30, l_{0}=0.85, H_{0}=\sqrt{0.9}$ and the cavity length is fixed to 3 . The fixed parameters imply a small signal gain of $30 \mathrm{~dB}$ in the gain fiber and a small signal transmittance of 0.15 for the SA. We use different filter bandwidths $\delta_{f}$ and the gain saturation energy $E_{\text {sat }}$ to investigate the impact of the spectral filtering. In the tuning process of $E_{\text {sat }}$, the pulse evolution shows complicated dynamics including multi-pulsing, bifurcations and chaos, and even a sudden jump of higher-energy, single pulse states. All of the observed dynamics and their interpretations are discussed below.

\section{A. Multi-pulsing transition}

The filter bandwidth $\delta_{f}$ is first set to 0.08 . This corresponds to a realistic filter bandwidth of $253 \mathrm{GHz}$ if other parameters are considered to be $\gamma=1 / \mathrm{W} / \mathrm{km}, \beta_{2}=-20 \mathrm{ps}^{2} / \mathrm{km}$ and $P_{\text {sat }}=$ $200 \mathrm{~W}$, which are very typical values for the mode-locked fiber laser cavities. Here we set $D_{3}=0$ to neglect the third order dispersion since the pulse bandwidth is not that broad.
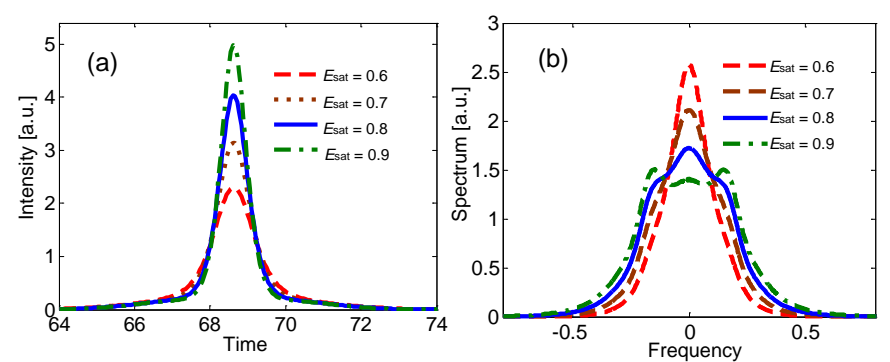

Fig. 3. (a) The pulse temporal profiles and (b) spectra when $E_{\text {sat }}$ is gradually increased from 0.6 to 0.9 with $\delta_{f}=0.08$.

Figure 3 shows the temporal waveforms and spectra of the solutions at the point $\mathrm{A}$ in the cavity with $E_{\text {sat }}$ varied from 0.6 to 0.9. With the increase of $E_{\text {sat }}$ up to 0.9 , there is always a single pulse observed in the cavity. It should be emphasized that with $E_{\text {sat }}=0.6$, the evolution always converged to the same pulse solution even when the simulations are started with different white noise seeds. Then $E_{\text {sat }}$ was gradually increased up to 0.9 in steps of 0.1. The simulation is iterated for enough round trips to guarantee that the pulse has been stabilized after each tuning step value of $E_{\text {sat. }}$ The pulse peak is gradually enhanced from 2.30 to 4.97 and the full width at half maximum (FWHM) is reduced from 1.31 to 0.79 . However, the pulse profile is kept similar and smooth in the tuning process. In contrast to the slight change of the temporal waveform, the variation of the spectrum is significant as shown in Fig. 3(b). With the increase of $E_{\text {sat }}$, the main peak of the spectrum decreases while there is formation and growth of the side wings because of nonlinear effects, e.g., self-phase modulation (SPM), in the gain fiber. Increasing values of $E_{\text {sat }}$ created pulses with increased bandwidth and large amount of side wings, which suffer from monotonically increased filtering loss from the Gaussian spectral filter. This is an energy dependent nonlinear loss. Accordingly, such filtering losses combined with the nonlinear loss due to the SA produce the non-monotonic transmission response (red curve) described in Fig. 1.

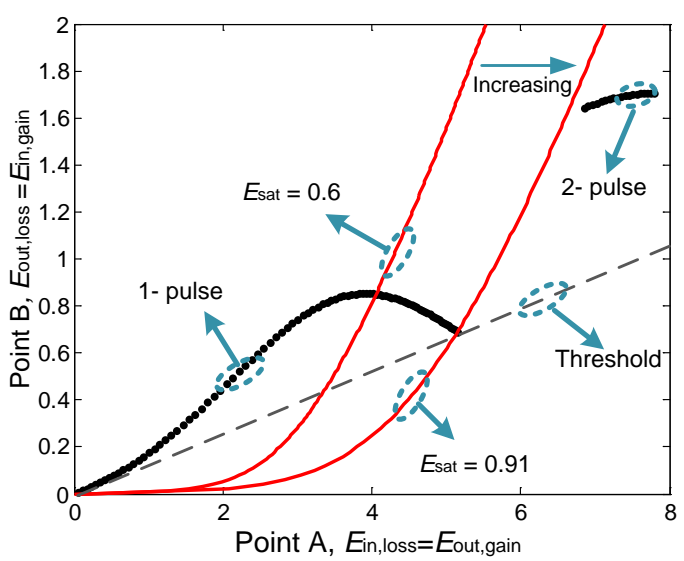

Fig. 4. The gain and loss curves in the laser cavity for $\delta_{f}=0.08$. The red solid curves are the $E_{\text {in,gain }}$ Vs $E_{\text {out,gain }}$ with $E_{\text {sat }}=0.6$ and 0.91 respectively. The black solid circles are the working points of combined nonlinear loss as $E_{\text {out,loss }}\left(E_{\text {in,loss }}\right)$. The gray dashed line is the threshold with a slope determined by the small signal loss.

To investigate the nonlinear response of the combined effects of SA and spectral filter, we monitor the energies at the points A and B in the cavity (Fig. 2). The response of the combined nonlinear loss is represented by the mapping of the energies at $\mathrm{A}\left(E_{\text {in,loss }}=E_{\text {out,gain }}\right)$ and $\mathrm{B}\left(E_{\text {in,gain }}=E_{\text {out,loss }}\right)$ with the final states for different $E_{\text {sat }}$, as shown by the black solid circles in Fig. 4. These solid circles also represent the input versus output of the gain fiber of the final states. The output energy of the gain fiber at point A, which are calculated by simply solving the $g_{n}$ expression in Eq. (6) for different input signal energies at point $\mathrm{B}$ (input of the gain fiber) and with different $E_{\text {sat, }}$ are represented as red solid curves. For clarity the red curves are drawn only for two values of $E_{\text {sat }}=0.6$ and 0.91 in Fig. 4 . The red curves pass through their corresponding final working points indicated by the black solid circles. The gray dashed threshold line, which is determined by the small signal loss, is drawn as the tangent of the black solid circles curve at the lowest small signal energy. Similar to the gain loss dynamics discussed in Refs. [9, 10], the final working point of the cavity climbs along the black solid circles with the increasing values of $E_{\text {sat }}$. With $E_{\text {sat }}=0.6$, the working point has just passed the peak. After passing the peak, if the working point drops to a value lower than the gray dashed threshold line, multi-pulsing transition is triggered in the cavity. For the parameters considered for this cavity, the working point reaches the threshold line when the $E_{\text {sat }}$ value is 0.91 .

Figure 5(a) shows the evolution of the pulse at point $B$ for the multi-pulsing transition when $E_{\text {sat }}$ is increased from 0.9 to 0.95 , where the dashed vertical line indicates the position of the tuning. With $E_{\text {sat }}=0.9$, a stable single pulse solution with a peak power of 0.192 and an FWHM of 3.37 is obtained. The temporal waveform of the signal is shown in Fig. 5(b). When $E_{\text {sat }}$ is tuned to 0.95 , the working point crosses the threshold line since the increased nonlinear effect expands the spectrum further and 
leads to higher filtering loss. Thus the pulse in the cavity will experience higher loss than the small signal. Several tiny pulses originating from the side lobes act as seeds for multi-pulsing. Initially all these tiny pulses get amplified before one of them grows to non-negligible level. Then the gain level in the cavity is again reduced by the saturation. Eventually one of the tiny pulses survives in addition to the original pulse, while the others are suppressed. The two formed pulses have an identical peak power of 0.184 and an FWHM of 4.10 as shown in Fig. 5(c). The pulses formed after the multi-pulsing transition have reduced peak power and increased pulse width compared to the former single pulse (Fig. 5(b)). Since the saturation energy of each pulse in the shared gain is halved, the effective gain curve for each pulse is shifted to left and the working point in the gain loss map of Fig. 4 is pushed to a point above the threshold line.
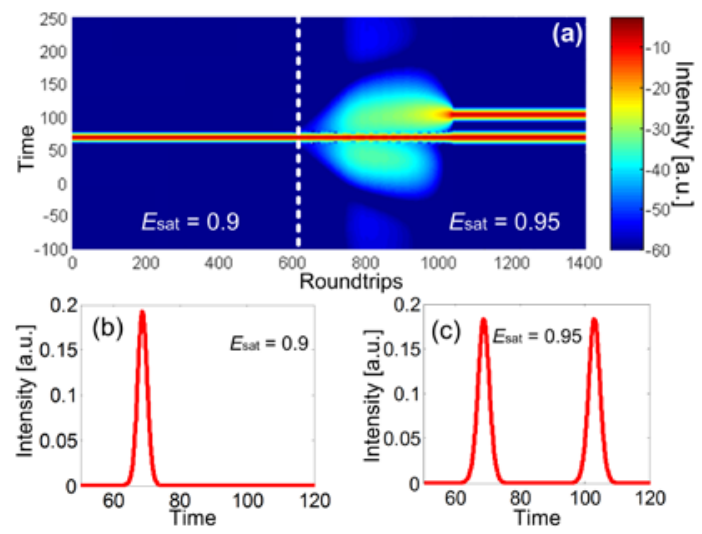

Fig. 5. (a) The pulse evolution when $E_{\text {sat }}$ is tuned from 0.9 to 0.95 with $\delta_{f}=0.08$. The temporal profiles of (b) single pulse and (c) two pulses in the cavity.

In Ref. [9], it was reported using the geometrical model that multi-pulsing could be observed in a cavity with an oscillating nonlinear loss but not with a monotonic loss. Here, with the extended geometrical model, we demonstrated that a laser cavity with a monotonic transmission response given by the SA could also have multi-pulsing transition dynamics due to the filtering loss of the spectral filter.

\section{B. Soliton breathing and single pulse higher energy transition}

If the spectrum of the pulse broadens self-similarly, continuously and monotonically for increasing values of $E_{\text {sat }}$, multi-pulsing transition is always observed for a given value of $E_{\text {sat }}$. But such ideal broadening of the spectrum is not the commonly observed dynamics in soliton fiber laser cavities. In case of weak gain, the pulse duration is adiabatically compressed as the peak of the pulse is increased [27, 28]. Adiabatic soliton lasers can be realized based on such adiabatic amplification [29]. However, such adiabatic amplification requires a relatively longer length of gain fiber that is detrimental for the lasers, e.g. the formation of Kelly sideband, which prevents the generation of higher energy pulses [29]. In most soliton fiber laser cavities, the pulse amplification is not adiabatic but similar to the soliton breathing (high order soliton evolutions) [27, 28, 30]. Spectral broadening and formation of side wings reported in Fig. 3(b) can be an indication of soliton breathing dynamics.

In the cavity, if the length of gain fiber is much shorter than the soliton period, the pulse evolution in the gain fiber can only engage the beginning spectral broadening section of the soliton breathing that leads to multi-pulsing as the $E_{\text {sat }}$ value is increased. If the length of the gain fiber is comparable or even longer than the soliton period, then the periodic higher order soliton evolution can bring a richer dynamics into the cavity. In passive fibers, the soliton period is calculated as $0.5 \pi L_{\mathrm{D}}$, where $L_{\mathrm{D}}$ is the dispersion length. Thus a soliton with shorter duration (wider spectral bandwidth) have a shorter soliton period. Although the soliton period in an active fiber medium is not the same as that of the passive fibers, the relation between the soliton period and the pulse duration (spectral bandwidth) should be qualitatively similar. Wider bandwidth spectral filter in the cavity can generate pulses with larger spectral width which corresponds to shorter soliton period.
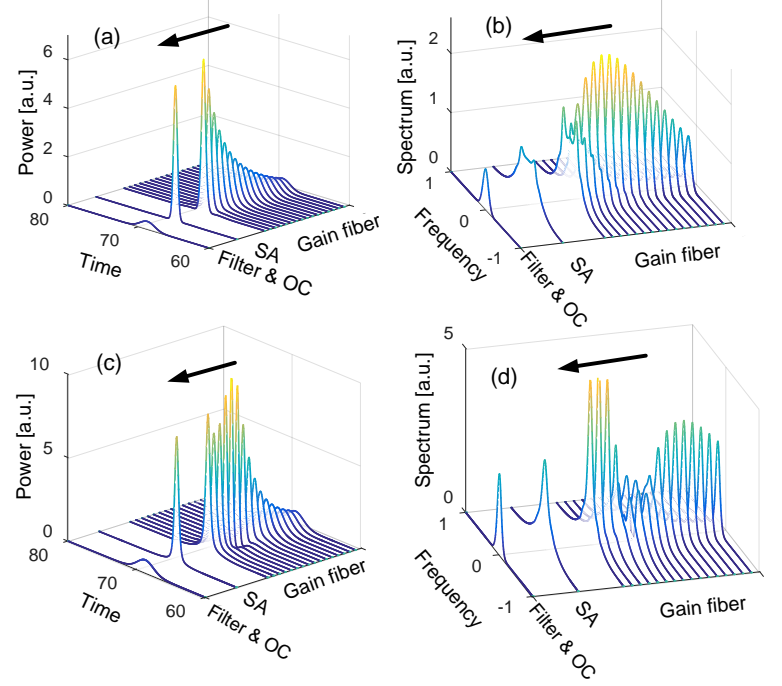

Fig. 6. The evolutions of (a), (c) pulse temporal and (b), (d) spectral waveforms in the laser cavity with (a), (b) $E_{\text {sat }}=0.8$, and (c), (d) $E_{\text {sat }}=1.0$, where $\delta_{f}=0.11$. Black arrows show the pulse propagation direction.

To investigate the dynamics of the high order soliton evolution, we increase the spectral filter bandwidth $\delta_{f}$ to 0.11 . Here we report two scenarios. First the gain fiber length is shorter compared to the pulse evolution so the spectrum does not evolve to its maximum. This case will accommodate only the beginning spectral broadening section in the evolution. The second case is when the gain fiber length is longer, hence the evolution would pass the first maximum point of the spectral bandwidth and then it would also evolve to the spectral narrowing section following the first maximum of its spectral bandwidth. The dynamics in such a laser cavity will be unstable when the evolution ends in the spectral narrowing section of soliton breathing, but eventually it will stabilize to a higher energy single pulse after going through an iterative pulse energy increasing dynamics. The evolution of this stable high-energy single pulse will end in the second spectral broadening section of soliton breathing in the gain fiber length. Hence one can observe a sudden increasing of the pulse energy.

Figures 6(a) and 6(b) respectively show the evolutions of the 
temporal and spectral waveforms of a single pulse with $E_{\text {sat }}=0.8$. In the gain fiber, the peak power of the pulse increases monotonically as shown in Fig. 6(a). The evolution in Fig. 6(b) shows that the spectrum of the pulse is broadening when propagating in the gain fiber and two side wings grow gradually in the spectrum. After the loss elements SA and filter (and OC), the side wings on the spectrum are completely suppressed with a large loss to the pulse energy. This is the case where the pulse evolution consists of only the beginning spectral broadening section in the gain fiber. If the filter gives a stronger filtering action in this case, multi-pulsing would be obtained due to the gain loss dynamics as mentioned above.

For the second case, if the output pulse of the gain fiber starts to narrow down its spectral bandwidth after the maximum bandwidth point, going through the SA and the filter makes the laser system unstable. But this instability is only transient before it dynamically attains a stable higher energy single pulse state. In this case the pulse spectrum after the gain fiber gets narrower and the loss given by the SA and filter in turn gets decreased. Thus the pulse coming out of the filter will have higher energy. This in-turn reduces the breathing period evolution and further narrows the output pulse spectrum of the gain fiber. Such iterative narrowing down of the pulse spectrum and increasing of the pulse energy (positive feedback) will continue until the pulse attains its second spectral broadening section. In this situation, higher energy pulses get wider spectra and experience larger filter loss (negative feedback). This will control and eventually stop the increasing of pulse energy. Thus the stable state is attained with a high-energy single pulse. In simulations such single pulse higher energy transition dynamics is observed when $E_{\text {sat }}$ is increased to 1.0. The evolutions of the temporal and spectral waveforms are shown in Figs. 6(c) and 6(d), respectively.

Figure 7(a) shows the geometrical mapping of such dynamics including the single pulse higher energy transition. The loss curve represented by the black solid circles is similar to that shown in Fig. 4 in most part but different when it reaches the threshold line. Nonlinear dynamics including periodic and chaotic states are observed in the region $0.8<E_{\text {sat }}<1.0$. To clearly show the regions that different states are found, we plot the pulse energy output from the filter $\left(E_{\text {out }}\right)$ versus $E_{\text {sat }}$ in Fig. 7(b). With the increase of $E_{\text {sat }}$, the signal has experienced single pulse, periodic solutions indicated by the bifurcation, chaotic solutions, another section of periodic solutions, and finally to single pulse high energy states. One can note the output energy of the high energy single pulse decreases with the increasing $E_{\text {sat. }}$ This is because these pulses evolution includes the second spectral broadening section as well in the gain fiber as mentioned above. Positive Lyapunov exponents are found for the chaotic states. The nonlinear dynamics are not new since similar dynamics have been observed and explained already in the literature $[9,10]$. Such a phenomenon has also been found in other models, e.g. the model in the framework of Ginzburg Landau equation [20]. Kutz also showed the periodic and chaotic states in the waveguide-array mode-locked fiber lasers $[18,19]$.
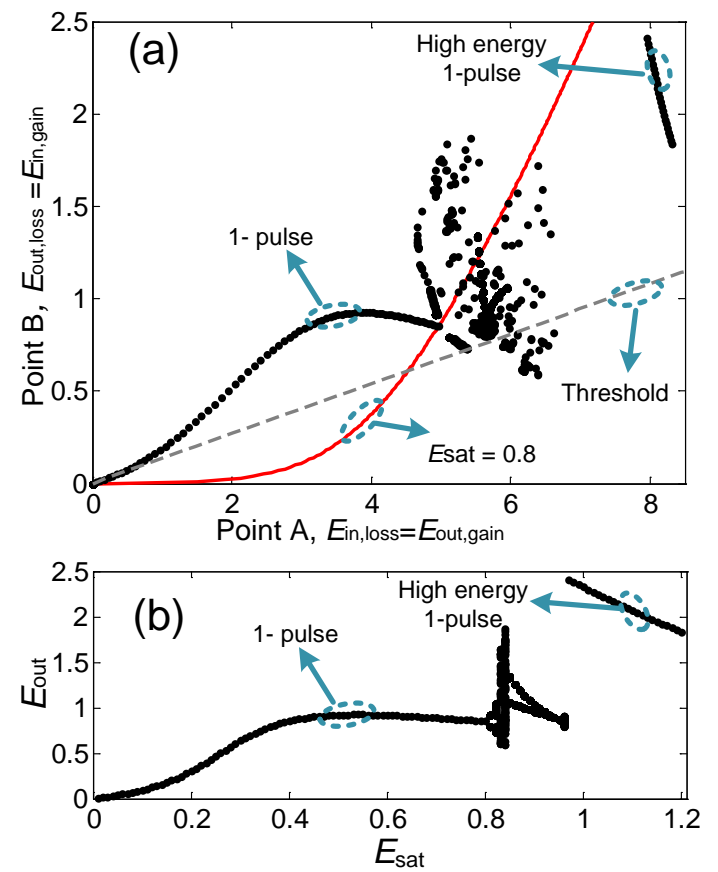

Fig. 7. (a) The geometrical mapping of gain and loss curves in the laser cavity for $\delta_{f}=0.11$. (b) The output energy of the filter $E_{\text {out }}$ versus $E_{\text {sat }}$.

\section{Working regions and transition boundaries}

In the above subsections $A$ and $B$, we have demonstrated different transition dynamics when the spectral filter bandwidth is set to different values. With narrower bandwidth, the pulse evolution in the gain fiber is rather simple and multi-pulsing is triggered by increasing values of $E_{\text {sat. }}$. When the spectral filter bandwidth is large, the rich soliton evolution leads to chaotic dynamics and finally to a stable single pulse higher energy transition.
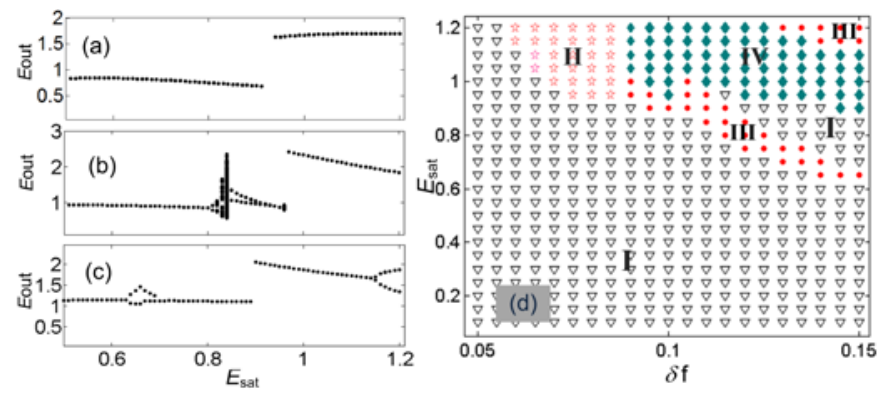

Fig. 8. The output energy of the filter $E_{\text {out }}$ versus $E_{\text {sat }}$ for (a) $\delta_{f}=0.08$, (b) $\delta_{f}=$ 0.11 and (c) $\delta_{f}=0.14$, (d) the laser dynamics as the function of filter bandwidth.

To investigate the impact of the spectral filter bandwidth, we show the regions, where different states are attained, and the typical transition processes in Fig. 8. Figures 8(a), 8(b) and 8(c) show three transition evolutions with $\delta_{f}=0.08,0.11$ and 0.14 , respectively. In Fig. 8(d), we varied both the filter bandwidth $\delta_{f}$ and the saturation energy $E_{\text {sat }}$ step by step and recorded the various operating states in the cavity. In region I indicated by the black triangles in Fig. 8(d), there is only single pulse state operating in the cavity. When $E_{\text {sat }}$ is increased, other states are observed depending on the values for $\delta_{f}$. For $\delta_{f} \leq 0.085$, the single pulse state either stays as it is or directly settles to two 
pulses multi-pulsing state (red stars) in the cavity without going through any complex dynamics. Region II with red stars indicates the parameter range of multi-pulsing state. The pulse energy evolution of $E_{\text {out }}$ (output of the filter) versus $E_{\text {sat }}$ with $\delta_{f}$ $=0.08$ is shown in Fig. 8(a) to demonstrate such transition. For a larger filter bandwidth $\delta_{f} \geq 0.09$, when $E_{\text {sat }}$ is increased, periodic and chaotic states are observed and they are shown as region III indicated by red solid circles in Fig. 8(d). After the periodic and chaotic states region III, when $E_{\text {sat }}$ is further increased, single pulse states are observed again. But these single pulse states are classified into two groups. In the region of $0.09 \leq \delta_{f} \leq 0.11$, the pulse jumps to a single pulse higher energy state that has been discussed in Fig. 7. This single pulse high-energy state is indicated using blue solid diamonds as region IV. Such transition is demonstrated by the pulse energy evolution of $E_{\text {out }}$ versus $E_{\text {sat }}$ with $\delta_{f}=0.11$ in Fig. 8(b). When $\delta_{f}$ if further increased to $\delta_{f} \geq 0.115$, the system will return to the original single pulse state I after the periodic and chaotic state III. Only when $E_{\text {sat }}$ is further increased, the single pulse gets evolved to the high-energy single pulse state IV directly. Figure 8(c) shows the pulse energy evolution of $E_{\text {out }}$ versus $E_{\text {sat }}$ for $\delta_{f}$ $=0.14$. In Fig. 8(c), periodic state is observed again after the high-energy single pulse state. Clearly, the high-energy single pulse state is not the final state since the nonlinear dynamics we have discussed in this paper is focused on the lower end of $E_{\text {sat }}$ values that lead to higher order soliton evolution. If $E_{\text {sat }}$ is further increased, periodic and chaotic states indicated by the region III at the right-top corner of Fig. 8(d) are attained.

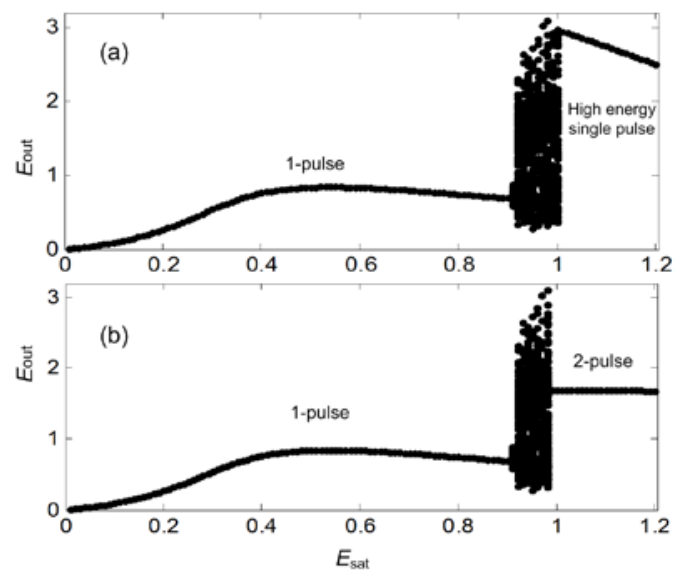

Fig. 9. The $E_{\text {out }}$ of the nonlinear loss elements as the function of $E_{\text {sat }}$ for $\delta_{f}=$ 0.09 . The transition dynamics shows that the chaotic state may lead to either a high energy single pulse state or a 2-pulse state due to its randomness.

Another point to be noted is the uncertain final state near the boundaries of different regions after the chaotic state. Due to the randomness of the chaotic state, further increase of the $E_{\text {sat }}$ would not guarantee a fixed final state after transition [9]. Figures 9(a) and 9(b) demonstrate two such situations when we gradually increase the $E_{\text {sat }}$ with $\delta_{f}=0.09$. After the chaotic region, a high-energy single pulse is obtained in Fig. 9(a) but a multipulsing state is attained in Fig. 9(b). This behavior is observed near the boundary between the multi-pulsing state and highenergy single pulse state where the thresholds to trigger either one of them are very close.

D. Impact of third order dispersion

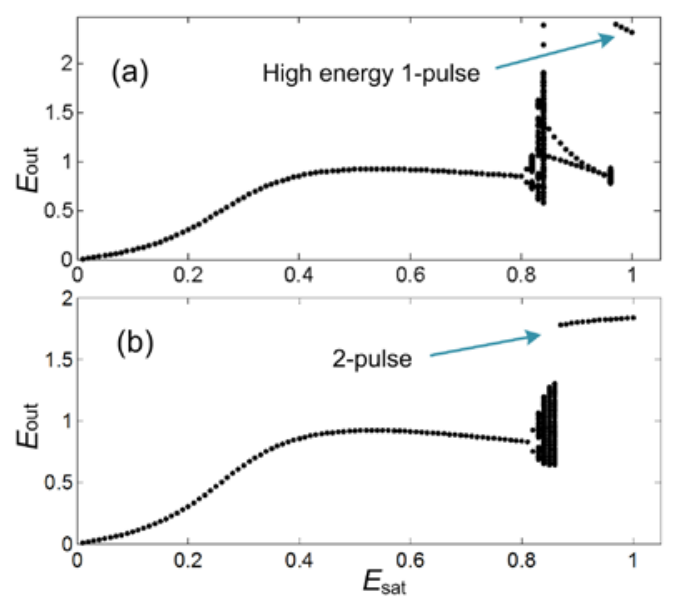

Fig. 10. $E_{\text {loss,out }}$ as the function of $E_{\text {sat }}$ when (a) $D_{3}=0.005$, and (b) $D_{3}=0.05$.

In all the above simulations, the third order dispersion $D_{3}$ is set to zero. For the high-energy single pulse state, we have shown that the pulse evolution in the gain fiber resembles the higher order soliton evolution. In the presence of third order dispersion, a higher order soliton could split into multiple fundamental solitons [30] and radiate dispersive wave if the spectrum covers the zero dispersion wavelength [31]. In the gain fiber, although the soliton evolution is modified by the soliton order increasing along the propagation due to the power amplification, the perturbation of third order dispersion to the pulse evolution is still similar to that in a passive fiber [27, 28]. It can be predicted that such perturbation will generate a seed to the multi-pulse transition and hence it can change the threshold for multi-pulsing.

We repeat the simulations shown in Fig. 7 with nonzero values for $D_{3}$ to investigate the impact of third order dispersion. The $E_{\text {out }}$ as the function of $E_{\text {sat }}$ with $D_{3}=0.005$ and 0.05 are shown in Figs. 10(a) and 10(b), respectively. When connecting with the real parameters, using the same set of parameters, $\gamma=$ $1 / \mathrm{W} / \mathrm{km}, \beta_{2}=-20 \mathrm{ps}^{2} / \mathrm{km}$ and $P_{\text {sat }}=200 \mathrm{~W}$, a fiber with $\beta_{3}=$ $0.1 \mathrm{ps}^{3} / \mathrm{km}$ will have $D_{3}=0.0026$ according to Eq. (6). The value of $D_{3}$ could be increased either by increasing the $P_{\text {sat }}$ of $\mathrm{SA}$, or decreasing the net dispersion $\beta_{2}$ of the cavity. The third order dispersion length is $\sim 200$ to 300 times of the gain fiber length for $D_{3}=0.005$, and $\sim 20$ to 30 times for $D_{3}=0.05$. In both cases, $E_{\text {out }}$ does not change significantly for $E_{\text {sat }}$ smaller than 0.8 . When $D_{3}=0.005$, the high-energy single pulse could still be obtained, but for $D_{3}=0.05$, multi-pulsing is triggered after the chaotic region and two pulses are observed in the cavity. With nonzero third order dispersion, the multi-pulsing threshold is lowered. Nonzero $D_{3}$ induces an asymmetry to the spectrum especially when the spectrum evolves to wider bandwidth. The asymmetry of the spectrum hence breaks the soliton recovering and radiates a small pulse from the main pulse, which becomes a large seed for the second pulse [27, 28, 31].

Besides the third order dispersion, other high order effects, e.g. self-steepening and Raman scattering, could also lead to 
similar dynamics to perturb the higher order soliton evolution. Similar to third order dispersion, they could introduce asymmetry to the soliton. Either acting alone or in combination, these effects can result in spectral-broadening [32] and pulse splitting [33, 34]. In this paper, we used the third order dispersion to demonstrate the impact of such perturbation that causes asymmetry to the pulse. In an accurate modeling of soliton fiber lasers to provide support to experiments, the necessity to include higher order effects depends on their magnitudes that depend on the spectral bandwidth and intensity of the intracavity pulse in operation.

\section{CONCLUSIONS}

The multi-pulsing instability is ubiquitous in mode-locked laser cavities. The geometrical model proposed in $[9,10]$ is an intuitive and simple approach to interpret the triggering of multi-pulsing transition. In this work, we proposed an extended geometrical model to include the effects of pulse shaping elements in the cavity. In laser cavities, the losses induced by spectral filtering can be combined with the saturable absorber as a combined nonlinear loss for such model. Based on this model, the impact of spectral filtering in anomalously dispersive soliton laser cavities has been investigated. With a narrow band spectral filter, the laser dynamics is found to be dominantly controlled by the gain-loss dynamics in the cavity which causes multi-pulsing. When the bandwidth of the spectral filter is large, the high order soliton evolution in the gain fiber plays a critical role in the laser dynamics. The system attains a high-energy single pulse state when the spectral filtering is applied at the spectral narrowing region of the evolution. Periodic and chaotic states are also observed in the laser dynamics. When nonzero third order dispersion is included, it is difficult to attain the high-energy single pulse state. The pulse splitting caused by third order dispersion can lead the cavity to multi-pulse state even if the threshold line in the gain loss dynamics is still not reached. It should be noted that the effect of spectral filtering in a realistic laser cavity could be more complex than the results reported in this work using the iterative model. The research studies presented in this paper are typical for soliton fiber lasers having net anomalous dispersion for the cavity. If multiple sections of fibers with different dispersion coefficients are introduced into the cavity, the pulse evolution can be different. The positions of the gain fiber and passive fiber will also affect the laser dynamics. The spectral filtering will also have a significant impact in determining the pulse dynamics when the pulse spectrum injected into the filter has a bandwidth comparable to that of the filter and depends on the pulse energy. The investigation results derived and reported in this work using the extended geometrical model are valid for any mode-locked fiber laser cavities similar to the schematic and the parametric conditions considered in the simulations. The extended geometrical model proposed in this work can be used as the basic model for the study of the impact of spectral filtering in laser cavities. This model can be appropriately modified to perform studies on laser cavities that are having different configurations compared to the basic one considered in this paper. To conclude, in this paper using an extended geometrical model we demonstrated that spectral filtering leads to strong nonlinear dynamics in a mode-locked fiber laser cavity, which can be utilized to understand and engineer the pulse dynamics in mode-locked soliton fiber lasers.

\section{REFERENCES}

[1] V. J. Matsas, D. J. Richardson, T. P. Newson, and D. N. Payne. "Characterization of a self-starting, passively mode-locked fiber ring laser that exploits nonlinear polarization evolution,” Opt. Lett., vol. 18, pp. 358-360, 1993.

[2] L. A. Gomes, L. Orsila, T. Jouhti, O. G. Okhotnikov, "Picosecond SESAM-based ytterbium mode-locked fiber lasers.” IEEE J. Sel. Top. Quantum Electron., vol. 10, pp. 129-136, 2004.

[3] D. J. Richardson, R. I. Laming, and D. N. Payne. "Pulse repetition-rates in a passive, self-starting, femtosecond soliton fibre laser.” Electron. Lett., vol. 27, pp. 1451-1453, 1991.

[4] M. Nakazawa, E. Yoshida, and Y. Kimura. "Generation of 98 fs optical pulses directly from an erbium-doped fibre ring laser at 1.57

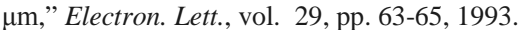

[5] A. B. Grudinin, D. J. Richardson, and D. N. Payne, "Energy quantization in figure eight fibre laser,” Electron. Lett., vol 28, pp. 67-68, 1992.

[6] M. E. Fermann and J. D. Minelly, "Cladding-pumped passive harmonically mode-locked fiber laser,” Opt. Lett. vol. 21, pp. 970-972 1996.

[7] C. Wang, W. Zhang, K. F. Lee, and K. M. Yoo, "Pulse splitting in a selfmode-locking Ti:sapphire laser,” Opt. Commun., vol. 137, pp. 89-92, 1997.

[8] H. Kitano and S. Kinoshita, "Stable multi-pulse generation from a selfmode-locked Ti:sapphire laser,” Opt. Commun., vol. 157, pp. 128-134, 1998.

[9] F. Li, P. K. A. Wai, and J. N. Kutz, "Geometrical description of the onset of multi-pulsing in mode-locked laser cavities,” J. Opt. Soc. Am. B, vol. 27, pp. 2068-2077, 2010.

[10] F. Li, E. Ding, J. N. Kutz, and P. K. A. Wai, "Dual transmission filters for enhanced energy in mode-locked fiber lasers,” Opt. Express, vol. 19, pp. 23408-23419, 2011.

[11] E. Ding, E. Shlizerman, and J. N. Kutz. "Generalized master equation for high-energy passive mode-locking: the sinusoidal Ginzburg-Landau equation,” IEEE J. Quantum Elect., vol. 47, pp. 705-714, 2011.

[12] X. Fu and J. N. Kutz, "High-energy mode-locked fiber lasers using multiple transmission filters and a genetic algorithm,” Opt. Express, vol. 21, pp. 6526-37, 2013.

[13] M. J. Lederer, B. Luther-Davies, H. H. Tan, C. Jagadish, N. N. Akhmediev, and J. M. Soto-Crespo, "Multipulse operation of a Ti:sapphire laser mode-locked by an ion-implanted semiconductor saturable-absorber mirror,” J. Opt. Soc. Am. B, vol. 16, pp. 895-904 1999.

[14] D. Li, D. Tang, L.M. Zhao, and D. Shen, "Mechanism of dissipativesoliton-resonance generation in passively mode-locked all-normaldispersion fiber lasers,” J. Lightwave Technol., vol. 33, pp. 3781-3787, 2015.

[15] A. Komarov, H. Leblond, and F. Sanchez, "Multi-stability and hysteresis phenomena in passively mode-locked fiber lasers,” Phys. Rev. A., vol. 71, pp. 053809, 2005.

[16] X. Zhang, F. Li, Z. Kang, J. Yuan, and P. K. A. Wai, "Spectral filtering induced multi-pulsing in mode-locked soliton lasers,” in Photonics and Fiber Technology 2016 (ACOFT, BGPP, NP), Sydney, Paper: JT4A.7, 2016.

[17] William H. Renninger, Andy Chong, and Frank W. Wise. "Self-similar pulse evolution in an all-normal-dispersion laser.” Physical Review A, vol. 82, pp. 021805, 2010.

[18] J. N. Kutz and B. Sandstede, "Theory of passive harmonic mode-locking using waveguide arrays,” Opt. Express, vol. 16, pp. 636-650, 2008.

[19] B. G. Bale, K. Kieu, J. N. Kutz, and F.Wise, "Transition dynamics for multi-pulsing in mode-locked lasers,” Opt. Express, vol.17, pp. 2313723146, 2009.

[20] J. M. Soto-Crespo, M. Grapinet, P. Grelu, and N. Akhmediev, "Bifurcations and multiple-period soliton pulsations in a passively mode-locked fiber laser,” Phys. Rev. E vol. 70, pp. 066612, 2004.

[21] F. Li, J. Yuan, Z. Kang, Q. Li, and P. K. A. Wai, "Modeling Frequency Comb Sources,” Nanophotonics, vol. 5, pp. 292-315, 2016.

[22] H. A. Haus, "Theory of mode locking with a fast saturable absorber," $J$. Appl. Phys., vol. 46, pp. 3049-3058, 1975. 
[23] F. X. Kartner, I. D. Jung, and U. Keller, "Soliton mode-locking with saturable absorbers,” IEEE J. Sel. Top. Quantum Electron., vol. 2, pp. 540-556, 1996.

[24] F. X. Kurtner, J. Aus der Au, and U. Keller. "Mode-locking with slow and fast saturable absorbers-what's the difference?” IEEE J. Sel. Top. Quantum Electron., vol. 4, pp. 159-168, 1998.

[25] S. K. Wang, S. Droste, L. C. Sinclair, I. Coddington, N. R. Newbury, T. F. Carruthers and C. R. Menyuk. "Wake mode sidebands and instability in mode-locked lasers with slow saturable absorbers,” Opt. Lett., vol. 42, pp. 2362-2365, 2017.

[26] S. K. Wang, A. Docherty, B. S. Marks, C. R. Menyuk. "Comparison of models of fast saturable absorption in passively mode-locked lasers,” Opt. Express., vol. 24, pp. 20228-20244, 2016.

[27] K. J. Blow, N. J. Doran, and D. Wood, "Generation and stabilization of short soliton pulses in the amplified nonlinear Schrödinger equation”, $J$. Opt. Soc. Am. B, vol. 5, pp. 381-391, 1988.

[28] K. J. Blow, N. J. Doran, and D. Wood, "Trapping of energy into solitary waves in amplified nonlinear dispersive systems.” Opt. Lett., vol. 12, pp. 1011-1013, 1987.

[29] Anastasia Bednyakova, and Sergei K. Turitsyn. “Adiabatic soliton laser,” Phys. Rev. Lett., vol. 114, pp. 113901, 2015.

[30] Agrawal, Govind P., Nonlinear fiber optics, $5^{\text {th }}$ edition, Academic press, 2013.

[31] P. K. A. Wai, C. R. Menyuk, Y. C. Lee, and H. H. Chen, "Nonlinear pulse propagation in the neighborhood of the zero-dispersion wavelength of monomode optical fibers," Opt Lett., vol. 11, pp. 464-466, 1986.

[32] J. Nathan Kutz, C. Lyngå, and B. J. Eggleton. "Enhanced supercontinuum generation through dispersion-management,” Opt. Express, vol. 13, pp. 3989-3998, 2005.

[33] Govind P. Agrawal, Nonlinear fiber optics. Academic press, 2007.

[34] K. Ohkuma, Yoshi H. Ichikawa, and Y. Abe. "Soliton propagation along optical fibers,” Opt. let., vol. 12, pp. 516-518, 1987

Xianting Zhang received the B.S. and M.E. degrees from Beijing University of Posts and Telecommunications, Beijing, China, in 2013 and 2016, respectively. Currently he is pursuing his Ph.D. degree at The Hong Kong Polytechnic University.

His research interests include fiber lasers, optical signal processing, and nonlinear fiber optics.

Feng Li received the B.S. and Ph.D. degrees from University of Science and Technology of China, Hefei, China, in 2001 and 2006, respectively.

After that, he joined the Hong Kong Polytechnic University as a postdoctoral fellow. Currently he is a research fellow the Hong Kong Polytechnic University. His research interests include fiber lasers, especially multi-wavelength lasers and mode-locked lasers, nonlinear fiber optics, supercontinuum generation, nonlinear silicon photonics, and nonlinear dynamics in optical devices and optical systems.

K. Nakkeeran received the B.Eng. degree from the Coimbatore Institute of Technology, Coimbatore, Tamil Nadu, India, in 1993, and the M.Technol. and Ph.D. degrees from Anna University, Chennai, Tamil Nadu, India, in 1995 and 1998, respectively. In 1999, he joined the Institute of Mathematical Sciences, Chennai, where he was a Postdoctoral Fellow for ten months. In 1999, he became a Research Associate with the Department of Physics, University of Burgundy, Dijon, France. In 2002, he became a Postdoctoral Fellow with the Department of Electronic and Information Engineering, The Hong Kong Polytechnic University. In 2005, he joined the School of Engineering, University of Aberdeen, Aberdeen, U.K. Currently he is a Senior Lecturer since 2011. His research interests include solitons, fiber lasers, modeling and simulations of optical devices, long-haul optical fiber communications, and nonlinear science. Dr. Nakkeeran is a member of the Optical Society of America and The Institution of Engineering and Technology (IET).

Jinhui Yuan received the B.S. and M.S. degrees from Yanshan University, Qinhuangdao, China, in 2005 and 2008, respectively. In 2011, he received the Ph.D. degree from Beijing University of Posts and Telecommunications (BUPT), Beijing, China.

Now he is with the BUPT as an associate professor. He is also a Hong Kong Scholar at the Photonics Research Centre, Department of Electronic and Information Engineering, The Hong Kong Polytechnic University. His current research interests include photonic crystal fibers, silicon waveguide, and optical fiber devices. He is the Members of IEEE and OSA.

Zhe Kang received the B.S. degree from Wuhan University of Technology, Wuhan, China, in 2006, the M.S. degree from Dalian Polytechnic University, Dalian, China, in 2012, and the Ph.D. degree from Beijing University of Posts and Telecommunications, Beijing, China, in 2015.

Currently he serves as the postdoctoral fellow in The Hong Kong Polytechnic University. His research interests include ultrafast nonlinear optics and nonlinear silicon photonics.

J. Nathan Kutz was awarded the B.S. in Physics and Mathematics from the University of Washington in 1990 and the PhD in Applied Mathematics from Northwestern University in 1994. Following postdoctoral fellowships at the Institute for Mathematics and its Applications (University of Minnesota, 1994-1995) and Princeton University (1995-1997), he joined the faculty of applied mathematics and served as Chair from 2007-2015.

P. K. A. Wai (SM'96) received the B.S. (Hons.) degree from the University of Hong Kong in 1981, and the M.S. and Ph.D. degrees from the University of Maryland, College Park, in 1985 and 1988, respectively.

In 1988, he joined Science Applications International Corporation, McLean, VA, where he was a Research Scientist involved with the Tethered Satellite System project. In 1990, he became a Research Associate with the Department of Physics, University of Maryland, College Park, and the Department of Electrical Engineering, University of Maryland, Baltimore County. In 1996, he joined the Department of Electronic and Information Engineering, The Hong Kong Polytechnic University. He became Chair Professor of Optical Communications in 2005. Currently he is the Vice President (Research Development). His research interests include soliton, fiber lasers, modeling and simulations of optical devices, longhaul optical fiber communications, all-optical packet switching, and network theories. He is an active contributor to the field of photonics and optical communications, having authored or coauthored over 300 international refereed publications. Currently he is an associate editor of Journal of Lightwave Technology.

Prof. P. K. A. Wai is the Fellows of the Institute of Electrical and Electronics Engineers (IEEE) and Optical Society of America (OSA). 